CU-TP-618

hep-th/9312074

\title{
Fermions in a Global Monopole Background
}

\author{
Hai Ren \\ Physics Department \\ Columbia University \\ New York, New York 10027
}

\begin{abstract}
We study the scattering of fermions in a "global monopole" background metric. This is the four-dimensional analogue of the scattering on a cone in three dimensions. The scattering amplitude is exactly obtained. We then study massless fermion-dyon systems in such a background metric. The density of the $S$-wave fermion condensate is found to be given by a constant times the flat space value of Callan and Rubakov.
\end{abstract}

This work was supported in part by the US Department of Energy 


\section{Scattering of Fermions}

We study the fermion scattering in a "global monopole" background metric (whose explicit form we will give below). This is one of the few examples where the scattering amplitude can be exactly obtained. It is the four-dimensional analogue of the scattering on a cone studied in [1]. The scattering of scalar particles in such a background is studied in [2].

First, we consider the general spherically symmetric metric

$$
d s^{2}=\beta^{2}(r) d t^{2}-\frac{1}{\alpha^{2}(r)} d r^{2}-R^{2}(r)\left(d \theta^{2}+\sin ^{2} \theta d \varphi^{2}\right)
$$

We choose the vierbein such that the fermion wave functions will be single-valued

$$
e_{a}^{\mu}=\left(\begin{array}{cccc}
\frac{1}{\beta} & 0 & 0 & 0 \\
0 & \alpha \sin \theta \cos \varphi & \frac{1}{R} \cos \theta \cos \varphi & -\frac{1}{R \sin \theta} \sin \varphi \\
0 & \alpha \sin \theta \sin \varphi & \frac{1}{R} \cos \theta \sin \varphi & \frac{1}{R \sin \theta} \cos \varphi \\
0 & \alpha \cos \theta & -\frac{1}{R} \sin \theta & 0
\end{array}\right)
$$

After standard manipulations, we arrive at the Dirac equation

$$
i\left[\frac{1}{\beta} \gamma^{0} \partial_{t}+i \boldsymbol{\gamma} \cdot \mathbf{p}^{(\alpha, R)}-\frac{1-\alpha R^{\prime}}{R} \boldsymbol{\gamma} \cdot \mathbf{n}+\alpha \frac{\beta^{\prime}}{2 \beta} \boldsymbol{\gamma} \cdot \mathbf{n}\right] \psi=M \psi
$$

where

$$
i \boldsymbol{\gamma} \cdot \mathbf{p}^{(\alpha, R)} \equiv \boldsymbol{\gamma} \cdot \mathbf{n} \alpha(r) \partial_{r}+\boldsymbol{\gamma} \cdot \partial_{\theta} \mathbf{n} \frac{1}{R(r)} \partial_{\theta}+\boldsymbol{\gamma} \cdot\left(\mathbf{n} \times \partial_{\theta} \mathbf{n}\right) \frac{1}{R(r) \sin \theta} \partial_{\varphi}
$$

$\mathbf{n} \equiv \hat{\mathbf{r}}$, and a prime denotes differentiation with respect to $r$.

Next we specialize to the global monopole background metric

$$
d s^{2}=d t^{2}-\frac{1}{\alpha^{2}} d r^{2}-r^{2}\left(d \theta^{2}+\sin ^{2} \theta d \varphi^{2}\right)
$$

where $\alpha \leq 1$ is a constant. It will be convenient to use the representation of the 
flat space $\gamma$-matrices

$$
\gamma^{0}=\left(\begin{array}{cc}
1 & 0 \\
0 & -1
\end{array}\right), \quad \gamma=\left(\begin{array}{cc}
0 & \boldsymbol{\sigma} \\
-\boldsymbol{\sigma} & 0
\end{array}\right)
$$

A complete set of solutions to the Dirac equation are of the form $\psi_{j m}^{(1)} e^{-i E t}$ and $\psi_{j m}^{(2)} e^{-i E t}$, with

$$
\psi_{j m}^{(1)}=\left[\begin{array}{c}
f(r) \phi_{j m}^{(1)} \\
g(r) \phi_{j m}^{(2)}
\end{array}\right], \quad \psi_{j m}^{(2)}=\left[\begin{array}{l}
f(r) \phi_{j m}^{(2)} \\
g(r) \phi_{j m}^{(1)}
\end{array}\right]
$$

where $\phi_{j m}^{(1)}$ and $\phi_{j m}^{(2)}$ are spinor spherical harmonics. For $\psi_{j m}^{(1)}$, the Dirac equation becomes

$$
\begin{aligned}
& (E-M) f=i\left(\partial_{r}+\frac{1+\nu}{r}\right) g \\
& (E+M) g=i\left(\partial_{r}+\frac{1-\nu}{r}\right) f
\end{aligned}
$$

where $\nu \equiv(j+1 / 2) / \alpha$, and we have redefined $E$ and $M$ by letting $E / \alpha \rightarrow$ $E, M / \alpha \rightarrow M$. The solution is given in terms of Bessel functions

$$
f=\frac{i k}{E-M} \frac{1}{\sqrt{k r}} J_{\nu-1 / 2}(k r), \quad g=\frac{1}{\sqrt{k r}} J_{\nu+1 / 2}(k r)
$$

where $k \equiv \sqrt{E^{2}-M^{2}}$. Similarly for $\psi_{j m}^{(2)}$, we have

$$
f=\frac{1}{\sqrt{k r}} J_{\nu+1 / 2}(k r), \quad g=\frac{i k}{E+M} \frac{1}{\sqrt{k r}} J_{\nu-1 / 2}(k r)
$$

We can now construct the scattering solution. The incident wave, with helicity +1 , has four components. However, when the upper two components are matched, the lower two components will automatically be matched. Also the overall normalization factor will cancel out at the end of the calculation. We may therefore only 
consider the upper two components of the incident wave

$$
\psi_{\text {in }}=e^{-i k z}\left(\begin{array}{l}
0 \\
1
\end{array}\right)
$$

whose large $r$ behavior is

$$
\begin{aligned}
e^{-i k z}\left(\begin{array}{c}
0 \\
1
\end{array}\right) \longrightarrow & -\frac{e^{i k r}}{i k r}\left(\frac{\pi}{2}\right)^{1 / 2} \sum_{j=1 / 2}^{\infty} \sqrt{2 j+1}(-1)^{j+1 / 2}\left(\phi_{j m}^{(1)}-\phi_{j m}^{(2)}\right) \\
& -\frac{e^{-i k r}}{i k r}\left(\frac{\pi}{2}\right)^{1 / 2} \sum_{j=1 / 2}^{\infty} \sqrt{2 j+1}\left(\phi_{j m}^{(1)}+\phi_{j m}^{(2)}\right)
\end{aligned}
$$

where $m \equiv-1 / 2$. It follows that the scattering solution is given by

$$
\psi=\pi \frac{E-M}{k} \sum_{j=1 / 2}^{\infty} e^{-i \pi \nu / 2} \sqrt{2 j+1}\left(\psi_{j m}^{(1)}+\frac{k}{E-M} \psi_{j m}^{(2)}\right)
$$

The outgoing wave, defined by $\psi=\psi_{\text {in }}+\psi_{\text {out }}$, is given for large $r$ by

$$
\psi_{\text {out }}=\frac{e^{i k r}}{i k r}\left(\frac{\pi}{2}\right)^{1 / 2} \sum_{j=1 / 2}^{\infty} \sqrt{2 j+1}\left[(-1)^{j+1 / 2}-e^{-i \pi \nu}\right]\left(\phi_{j m}^{(1)}-\phi_{j m}^{(2)}\right)
$$

where $\nu \equiv(j+1 / 2) / \alpha$. The scattering amplitude $f(\theta)$ is determined form

$$
\psi_{\text {out }}=f(\theta) \frac{e^{i k r}}{r} \chi
$$

where

$$
\chi \equiv \frac{1}{[2(1-\cos \theta)]^{1 / 2}}\left(\begin{array}{c}
\sin \theta e^{-i \varphi} \\
1-\cos \theta
\end{array}\right)
$$

Letting $\theta \rightarrow \pi-\theta, \theta$ now representing the scattering angle, we obtain the scattering amplitude

$$
f(\theta)=\frac{i}{2 k \cos \theta / 2} \sum_{n=1}^{\infty} n\left(1-e^{-i n \omega \pi}\right)\left[P_{n-1}(\cos \theta)+P_{n}(\cos \theta)\right]
$$

where $\omega \equiv(1 / \alpha)-1$, and $P_{n}$ is the Legendre polynomial. The summation can be naively (in the sense that we do not consider regularizing possibly ill-defined sums) 
carried out to obtain

$$
f(\theta)=\frac{i}{k} \delta(1-\cos \theta)+\frac{\sin \omega \pi / 2 \cos \theta / 2}{\sqrt{2} k(\cos \omega \pi-\cos \theta)^{3 / 2}}
$$

with the understanding that the $\delta$-function integrates to one rather than a half.

Note that $f(\theta)$ diverges at $\theta=0, \omega \pi$. This is similar to what happens in $2+1$ dimensions and is due to the long-range nature of the "interaction" - since the metric is not asymptotically flat [1]. Moreover, $f(\theta)$ does not vanish in the "nointeraction" limit, $\omega \rightarrow 0$. This also happens in the case of non-relativistic Coulomb scattering, where one is also left with a $\delta$-function term in the no-interaction limit.

\section{Fermion-Dyon Systems}

First, we study fermion-dyon systems in the general spherically symmetric background metric, the Euclidean version of Eq.(1). We consider a $S U(2)$ model with two massless left-handed doublets. The action is given by $S=S_{A}+S_{A, \psi}$ with

$$
\begin{aligned}
S_{A} & =\int \sqrt{g} d^{4} x\left[\frac{1}{2 e^{2}} \operatorname{tr} F_{\mu \nu}^{2}-M_{m o n}\right] \\
S_{A, \psi} & =i \sum_{s=1}^{2} \int \sqrt{g} d^{4} x \bar{\psi}_{L}^{(s)} \gamma^{\mu}\left(\partial_{\mu}-\Gamma_{\mu}+A_{\mu}\right) \psi_{L}^{(s)}
\end{aligned}
$$

where $s=1,2$ is the flavor index, $\Gamma_{\mu}$ is the spin connection, and $M_{m o n}$ is a constant. We have neglected the Higgs field contribution to the action. Spherically symmetric $S U(2)$ gauge fields are taken to be

$$
\begin{aligned}
& A_{0}(r, t)=-i \boldsymbol{\tau} \cdot \mathbf{n} a_{0}(r, t) \\
& A_{i}(r, t)=-i \boldsymbol{\tau} \cdot \mathbf{n} n_{i} \frac{a_{1}(r, t)}{\alpha(r) \beta(r)}-i(\mathbf{n} \times \boldsymbol{\tau})_{i} \frac{1-F(r)}{2 R(r)}
\end{aligned}
$$

In such a model, the singlet chiral number currents are anomalous

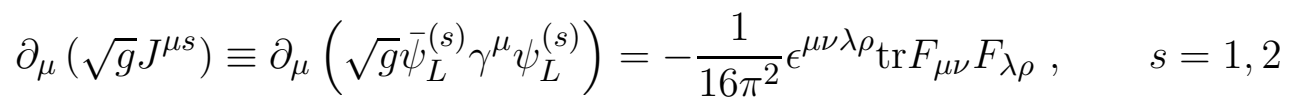


Therefore the gauge invariant chiral fermion number operators

$$
N^{(s)} \equiv \int d^{3} x \sqrt{g} J^{0 s}, \quad s=1,2
$$

are not conserved.

We will use the representation of the flat space $\gamma$-matrices

$$
\gamma^{0}=\left(\begin{array}{cc}
0 & -i \\
i & 0
\end{array}\right), \quad \gamma=\left(\begin{array}{cc}
0 & \boldsymbol{\sigma} \\
\boldsymbol{\sigma} & 0
\end{array}\right), \quad \gamma^{5}=\left(\begin{array}{cc}
1 & 0 \\
0 & -1
\end{array}\right)
$$

For the two component, left-handed spinors $\psi_{-}^{(s)}$, the Dirac equation reduces to

$$
\begin{aligned}
0= & {\left[\frac{1}{\beta} \partial_{t}-\boldsymbol{\sigma} \cdot \mathbf{p}^{(\alpha, R)}-i \frac{1-\alpha R^{\prime}}{R} \boldsymbol{\sigma} \cdot \mathbf{n}+i \alpha \frac{\beta^{\prime}}{2 \beta} \boldsymbol{\sigma} \cdot \mathbf{n}\right.} \\
& \left.\quad-i \frac{1}{\beta} a_{0} \boldsymbol{\tau} \cdot \mathbf{n}+\frac{1}{\beta} a_{1} \boldsymbol{\sigma} \cdot \mathbf{n} \boldsymbol{\tau} \cdot \mathbf{n}+\frac{1-F(r)}{2 R}(\boldsymbol{\sigma} \times \mathbf{n}) \cdot \boldsymbol{\tau}\right] \psi_{-}^{(s)}
\end{aligned}
$$

where $\boldsymbol{\sigma} \cdot \mathbf{p}^{(\alpha, R)}$ is given by Eq.(4) with $\boldsymbol{\sigma}$ replacing $\boldsymbol{\gamma}$. Spherically symmetric $S U(2)$ isospinor fermion fields are of the form [3]

$$
\psi_{-}^{(s)}(r, t)_{i n}=\frac{1}{\sqrt{8 \pi \beta R^{2}}}\left[\chi_{1}^{(s)}(r, t)-i \chi_{2}^{(s)}(r, t) \boldsymbol{\sigma} \cdot \mathbf{n}\right]_{i m}\left(i \tau^{2}\right)_{m n}
$$

where $i$ is the spin index and $n$ the isospin index. Introducing

$$
\chi^{(s)} \equiv\left(\begin{array}{c}
\chi_{1}^{(s)} \\
\chi_{2}^{(s)}
\end{array}\right)
$$

one finds that, in the $S$-wave approximation, the currents $J^{\mu s}$ have the following reductions

$$
J^{t s}=-\frac{1}{4 \pi \beta^{2} R^{2}} \bar{\chi}^{(s)} \tilde{\gamma}^{0} \chi^{(s)}, \quad J^{r s}=-\frac{\alpha}{4 \pi \beta R^{2}} \bar{\chi}^{(s)} \tilde{\gamma}^{1} \chi^{(s)}
$$

The original model can be reduced to the two dimensional model described by the 
action $S=S_{A}+S_{A, \psi}$ with

$$
\begin{aligned}
S_{A} & =\frac{4 \pi}{e^{2}} \int d x d t\left[\left(\partial_{x} a_{0}-\partial_{t} a_{1}\right)^{2} \frac{R^{2}(r)}{\beta^{2}(r)}\right] \\
S_{A, \psi} & =\sum_{s=1}^{2} \int d x d t \bar{\chi}^{(s)} \tilde{\gamma}^{\alpha}\left(i \partial_{\alpha}-\tilde{\gamma}^{5} a_{\alpha}\right) \chi^{(s)} \equiv i \sum_{s=1}^{2} \int d x d t \chi^{(s) \dagger} \mathcal{D} \chi^{(s)}
\end{aligned}
$$

where $\tilde{\gamma}^{0}=\sigma^{3}, \tilde{\gamma}^{1}=\sigma^{1}, \tilde{\gamma}^{5}=-i \tilde{\gamma}^{0} \tilde{\gamma}^{1}=\sigma^{2}$ and $s$ is the flavor index. We have taken the zero magnetic monopole size limit and introduced

$$
d r=\alpha(r) \beta(r) d x
$$

We see that the fermion action can always be reduced to a two-dimensional, flat space form. This remains true for massive fermions. Thus one can always integrate out the fermion fields (for massless fermions) or bosonize the action. These can be used to further understand systems with fermions and magnetically charged black holes. Such problems were recently introduced and studied in [4].

Next we specialize to the global monopole background metric and calculate the density of the fermion condensate, $\left\langle\psi_{-}^{(1) c \dagger} \psi_{-}^{(2)}>^{\text {mon }}\right.$, where 1,2 are flavor indices and $\psi_{-}^{(s) c}$ is the fermion number conjugation of $\psi_{-}^{(s)}$

$$
\left(\psi_{-}^{(s)}\right)_{i n}^{c} \equiv\left(i \sigma^{2}\right)_{i j}\left(i \tau^{2}\right)_{n m}\left(\psi_{-}^{(s)}\right)_{j m}^{*}
$$

One can verify that $\psi_{-}^{(s) c}$ satisfies the Dirac equation, Eq.(24), provided $\psi_{-}^{(s)}$ satisfies the Dirac equation. Using the Ansatz Eq.(25) we obtain

$$
\psi_{-}^{(1) c \dagger} \psi_{-}^{(2)}=\frac{1}{4 \pi r^{2}}\left(\chi_{1}^{(1)} \chi_{1}^{(2)}+\chi_{2}^{(1)} \chi_{2}^{(2)}\right) \equiv \frac{1}{4 \pi r^{2}} f(x, t)
$$

To calculate $<f(x, t)>^{\text {mon }}$, one uses the cluster property

$$
\lim _{\left|t_{1}-t_{2}\right| \rightarrow \infty}<f\left(x_{1} t_{1}\right) f^{\dagger}\left(x_{2} t_{2}\right)>^{m o n}=<f\left(x_{1} t_{1}\right)>^{m o n}<f^{\dagger}\left(x_{2} t_{2}\right)>^{m o n}
$$

The remaining computation closely follows that of Callan and Rubakov [5]. It is to some extent similar to that used in studying the massless Schwinger model [6]. 
The first step is to find the exact fermion propagator, which satisfies

$$
\left[\partial_{t}+i \sigma^{2} a_{0}+i \sigma^{2}\left(\partial_{x}+i \sigma^{2} a_{1}\right)\right] G\left(x t, x^{\prime} t^{\prime}\right)=\delta\left(x-x^{\prime}\right) \delta\left(t-t^{\prime}\right)
$$

and the "bag" boundary condition [5]

$$
\left(1-\sigma^{3}\right) G\left(0 t, x^{\prime} t^{\prime}\right)=0
$$

It can be solved by the Ansatz [6]

$$
G\left(x t, x^{\prime} t^{\prime}\right)=\exp [\phi(x, t)] G_{0}\left(x t, x^{\prime} t^{\prime}\right) \exp \left[-\phi\left(x^{\prime}, t^{\prime}\right)\right]
$$

where $G_{0}$ is the free fermion propagator satisfying the bag boundary condition, and is given by

$$
G_{0}\left(x t, x^{\prime} t^{\prime}\right)=\left(\partial_{t}-i \sigma^{2} \partial_{x}\right)\left[\Delta\left(x-x^{\prime}, t-t^{\prime}\right)+\Delta\left(x+x^{\prime}, t-t^{\prime}\right) \sigma^{3}\right]
$$

and $\phi$ is found to be

$$
\phi(x, t)=\zeta(x, t)-i \sigma^{2} \gamma(x, t)
$$

with

$$
\begin{aligned}
\zeta(x, t)=\partial_{t} & \int d x^{\prime} d t^{\prime}\left[\Delta\left(x-x^{\prime}, t-t^{\prime}\right)+\Delta\left(x+x^{\prime}, t-t^{\prime}\right)\right] a_{1}\left(x^{\prime}, t^{\prime}\right) \\
& -\partial_{x} \int d x^{\prime} d t^{\prime}\left[\Delta\left(x-x^{\prime}, t-t^{\prime}\right)-\Delta\left(x+x^{\prime}, t-t^{\prime}\right)\right] a_{0}\left(x^{\prime}, t^{\prime}\right) \\
\gamma(x, t)=\partial_{x} & \int d x^{\prime} d t^{\prime}\left[\Delta\left(x-x^{\prime}, t-t^{\prime}\right)+\Delta\left(x+x^{\prime}, t-t^{\prime}\right)\right] a_{1}\left(x^{\prime}, t^{\prime}\right) \\
& +\partial_{t} \int d x^{\prime} d t^{\prime}\left[\Delta\left(x-x^{\prime}, t-t^{\prime}\right)-\Delta\left(x+x^{\prime}, t-t^{\prime}\right)\right] a_{0}\left(x^{\prime}, t^{\prime}\right)
\end{aligned}
$$

where $\Delta$ is the free scalar propagator

$$
\Delta(x, t)=\frac{1}{4 \pi} \ln \mu^{2}\left(x^{2}+t^{2}\right)
$$

The choice of the boundary terms in Eq.(38) is to ensure that $\partial_{x} \zeta(0, t)=0$ and $\gamma(0, t)=0$, so that the boundary condition Eq.(34) can be satisfied. 
Under a gauge transformation $a_{0} \rightarrow a_{0}-\partial_{t} \beta, a_{1} \rightarrow a_{1}-\partial_{x} \beta$, one can verify that $\zeta \rightarrow \zeta, \gamma \rightarrow \gamma-\beta$. Therefore the boundary condition does not change the gauge transformation properties of the exact fermion propagator. The gauge invariant expression for $\operatorname{tr} G(\xi, \xi)$ is

$$
\operatorname{tr} G(\xi, \xi)=\left.\operatorname{tr}\left[G\left(\xi, \xi^{\prime}\right) \exp \left(i \sigma^{2} \int_{\xi^{\prime}}^{\xi} d \tilde{\xi}^{\alpha} a_{\alpha}(\tilde{\xi})\right)\right]\right|_{\xi^{\prime} \rightarrow \xi}=\frac{1}{\pi} \partial_{t} \zeta
$$

where we have introduced $\left(\xi^{0}, \xi^{1}\right) \equiv(t, x)$, and the approach of $\xi^{\prime}$ to $\xi$ is performed from a spatial direction.

One may calculate the induced vacuum currents

$$
\begin{aligned}
& j^{\alpha s}=<\bar{\chi}^{(s)} \tilde{\gamma}^{\alpha} \chi^{(s)}>\left.\right|_{A}=\operatorname{tr}\left[\tilde{\gamma}^{0} \tilde{\gamma}^{\alpha} G(\xi, \xi)\right]=\frac{1}{\pi}\left(\partial_{t} \zeta, \partial_{x} \zeta\right) \\
& j_{5}^{\alpha s}=<\bar{\chi}^{(s)} \tilde{\gamma}^{5} \tilde{\gamma}^{\alpha} \chi^{(s)}>\left.\right|_{A}=\operatorname{tr}\left[\tilde{\gamma}^{0} \tilde{\gamma}^{5} \tilde{\gamma}^{\alpha} G(\xi, \xi)\right]=\frac{i}{\pi}\left(\partial_{x} \zeta,-\partial_{t} \zeta\right)
\end{aligned}
$$

Therefore the axial-vector current is conserved, $\partial_{\alpha} j_{5}^{\alpha s}=0$, while the vector current possesses an anomaly

$$
\partial_{\alpha} j^{\alpha s}=\frac{1}{\pi}\left(\partial_{t}^{2}+\partial_{x}^{2}\right) \zeta=-\frac{1}{\pi}\left(\partial_{x} a_{0}-\partial_{t} a_{1}\right)
$$

which agrees with Eqs.(21) and (27).

The determinant of an operator $\mathcal{O}$, depending on some parameter $\lambda$, may be computed from the Green's function $G\left(\xi, \xi^{\prime}\right)$ associated with $\mathcal{O}$ as follows

$$
\frac{\partial}{\partial \lambda} \ln \operatorname{Det} \mathcal{O}=\operatorname{tr} \frac{\partial \mathcal{O}}{\partial \lambda} \int d \xi G(\xi, \xi)
$$

Using this, we obtain the fermion determinant

$$
\ln \operatorname{Det} \mathcal{D}=\frac{1}{2 \pi} \int d x d t \zeta \square \zeta=\ln \operatorname{Det} i \mathcal{D}
$$

where $\square \equiv\left(\partial_{t}^{2}+\partial_{x}^{2}\right)$. The effective action, obtained by integrating out the fermion 
fields, is thus

$$
S_{e f f}(\zeta)=S_{A}(\zeta)-2 \ln \operatorname{Det} i \mathcal{D}=\frac{1}{2} \int d x d t \zeta(x, t) L_{x t} \zeta(x, t)
$$

where

$$
L_{x t} \equiv \frac{2}{\pi}\left[-\square+\frac{4 \pi^{2} \alpha^{2}}{e^{2}} \square x^{2} \square\right]
$$

The Green's function associated with $L_{x t}$, satisfying $\partial_{x} \mathcal{P}\left(0 t, x^{\prime} t^{\prime}\right)=0$, is given by

$$
\mathcal{P}\left(x t, x^{\prime} t^{\prime}\right)=\frac{\pi}{2}\left[D_{e^{2} / 4 \pi^{2} \alpha^{2}}\left(x t, x^{\prime} t^{\prime}\right)-\Delta\left(x-x^{\prime}, t-t^{\prime}\right)-\Delta\left(x+x^{\prime}, t-t^{\prime}\right)\right]
$$

where $D_{\kappa}$ satisfies [5]

$$
\left(\partial_{t}^{2}+\partial_{x}^{2}-\frac{\kappa}{x^{2}}\right) D_{\kappa}\left(x t, x^{\prime} t^{\prime}\right)=\delta\left(x-x^{\prime}\right) \delta\left(t-t^{\prime}\right)
$$

with $\kappa=e^{2} / 4 \pi^{2} \alpha^{2}$. Eq.(48) can be solved by a Fourier transform with respect to time. One finds

$$
\left(-\omega^{2}+\partial_{x}^{2}-\frac{\kappa}{x^{2}}\right) \widetilde{D}_{\kappa}\left(x, x^{\prime}, \omega\right)=\frac{1}{2 \pi} \delta\left(x-x^{\prime}\right)
$$

Hence

$$
\widetilde{D}_{\kappa}\left(x, x^{\prime}, \omega\right)= \begin{cases}-\frac{\sqrt{x x^{\prime}}}{2 \pi} I_{\nu}(|\omega| x) K_{\nu}\left(|\omega| x^{\prime}\right), & x<x^{\prime} \\ -\frac{\sqrt{x x^{\prime}}}{2 \pi} I_{\nu}\left(|\omega| x^{\prime}\right) K_{\nu}(|\omega| x), & x>x^{\prime}\end{cases}
$$

where $\nu \equiv \sqrt{\kappa+1 / 4}$ and $I, K$ are modified Bessel functions. One can then Fourier transform back [7] to obtain

$$
D_{\kappa}\left(x t, x^{\prime} t^{\prime}\right)=-\frac{1}{2 \pi} Q_{\nu-1 / 2}\left[1+\frac{\left(x-x^{\prime}\right)^{2}+\left(t-t^{\prime}\right)^{2}}{2 x x^{\prime}}\right]
$$

where $Q$ is the Legendre function. 
The fermionic Green's functions in the presence of the magnetic monopole are given by

$$
\begin{aligned}
& <\chi\left(x_{1} t_{1}\right) \cdots \chi\left(x_{N} t_{N}\right) \chi^{\dagger}\left(x_{1}^{\prime} t_{1}^{\prime}\right) \cdots \chi^{\dagger}\left(x_{N}^{\prime} t_{N}^{\prime}\right)>^{m o n} \\
& \quad=\int D \zeta e^{-S_{e f f}(\zeta)}<\chi\left(x_{1} t_{1}\right) \cdots \chi\left(x_{N} t_{N}\right) \chi^{\dagger}\left(x_{1}^{\prime} t_{1}^{\prime}\right) \cdots \chi^{\dagger}\left(x_{N}^{\prime} t_{N}^{\prime}\right)>\left.\right|_{A}
\end{aligned}
$$

To calculate the density of condensate, one obtains $<f\left(x_{1} t_{1}\right) f^{\dagger}\left(x_{2} t_{2}\right)>^{\text {mon }}$ from

$$
\begin{gathered}
<f\left(x_{1} t_{1}\right) f^{\dagger}\left(x_{2} t_{2}\right)>\left.\right|_{A}=<\chi_{\alpha}^{(1)}\left(x_{1} t_{1}\right) \chi_{\alpha}^{(2)}\left(x_{1} t_{1}\right) \chi_{\beta}^{(1) \dagger}\left(x_{2} t_{2}\right) \chi_{\beta}^{(2) \dagger}\left(x_{2} t_{2}\right)>\left.\right|_{A} \\
=\operatorname{tr}\left[G_{0}\left(x_{1} t_{1}, x_{2} t_{2}\right) G_{0}^{\mathrm{T}}\left(x_{1} t_{1}, x_{2} t_{2}\right)\right] \exp \left[2 \zeta\left(x_{1}, t_{1}\right)-2 \zeta\left(x_{2}, t_{2}\right)\right]
\end{gathered}
$$

where we have used Wick's theorem and Eqs.(35), (37). Therefore

$$
\begin{aligned}
&<f\left(x_{1} t_{1}\right) f^{\dagger}\left(x_{2} t_{2}\right)>^{m o n}=\operatorname{tr}\left[G_{0}\left(x_{1} t_{1}, x_{2} t_{2}\right) G_{0}^{\mathrm{T}}\left(x_{1} t_{1}, x_{2} t_{2}\right)\right] \\
& \times \int D \zeta \exp \left[-S_{\text {eff }}(\zeta)+2 \zeta\left(x_{1}, t_{1}\right)-2 \zeta\left(x_{2}, t_{2}\right)\right] \\
&= \operatorname{tr}\left[G_{0}\left(x_{1} t_{1}, x_{2} t_{2}\right) G_{0}^{\mathrm{T}}\left(x_{1} t_{1}, x_{2} t_{2}\right)\right] \\
& \times \exp \left[-4 \mathcal{P}\left(x_{1} t_{1}, x_{2} t_{2}\right)+2 \mathcal{P}\left(x_{1} t_{1}, x_{1} t_{1}\right)+2 \mathcal{P}\left(x_{2} t_{2}, x_{2} t_{2}\right)\right] \\
&=\frac{1}{4 \pi^{2} x_{1} x_{2}}\left[1+O\left(\frac{e^{2}}{\alpha^{2}}\right)\right]
\end{aligned}
$$

where we have used the saddle-point method and taken the limit $\left|t_{1}-t_{2}\right| \rightarrow \infty$ on the last line, and used properties of the Legendre function [5]. According to the cluster property, Eq.(54) implies

$$
<f(x, t)>^{m o n}=\frac{e^{i \theta}}{2 \pi x}\left[1+O\left(\frac{e^{2}}{\alpha^{2}}\right)\right]
$$

where $\theta$ is an arbitrary phase. Recall that $x \equiv r / \alpha$, we have thus found that the density of the fermion number condensate is $\alpha$ times the flat space value. Therefore the amount of condensate from radius $r$ to $r+d r$ is the same as in flat space.

Acknowledgements:

I thank Professor Erick Weinberg for helpful discussions and valuable comments on the manuscript, Professor Roman Jackiw for a critical reading of the paper. 


\section{REFERENCES}

1. S.Deser and R.Jackiw, Comm.Math.Phys. 118, 495 (1988); P.Gerbert and R.Jackiw, ibid. 124, 229 (1989).

2. P.Mazur and J.Papavassiliou, Phys.Rev.D44, 1317 (1991).

3. R.Jackiw and C.Rebbi, Phys.Rev.D13, 3398 (1976).

4. C.Holzhey and F.Wilczek, Nucl.Phys.B380, 447 (1992);

M.G.Alford and A.Strominger, Phys.Rev.Lett.69, 563 (1992).

5. C.G.Callan, Jr., Phys.Rev.D25, 2141 (1982); V.A.Rubakov, Nucl.Phys. B203, 311 (1982).

6. N.K.Nielsen and B.Schroer, Nucl.Phys. B120, 62 (1977); J.Schwinger, Phys.Rev. 128, 2425 (1962).

7. I.S.Gradshteyn and I.M.Ryzhik, Table of Integrals, Series, and Products, (Academic, New York, 1980) page 732. 